\title{
Concomitant euthyroid Graves' ophthalmopathy and isolated ocular myasthenia gravis
}

\author{
Hussein Raef, Morrissa Ladinsky and Ridha Arem
}

Division of Endocrinology and Metabolism, Baylor College of Medicine, One Baylor Plaza, Houston, TX
77030, USA

Summary: A 44 year old diabetic woman presented with diplopia and bilateral ptosis and mild exophthalmos. The patient was clinically euthyroid, the baseline thyroid function tests were normal, but the thyroid stimulating hormone response to thyrotrophin releasing hormone was flat. Computed tomographic scan and magnetic resonance imaging of the orbits showed left medial and inferior rectus muscle thickening, more prominent on the left side, consistent with Graves' disease. The tensilon stimulation test resulted in resolution of the ptosis and partial improvement of the ophthalmoplegia. The single fibre electromyography was consistent with a defect in neuromuscular transmission. However, forced duction test was normal and anti-acetylcholine receptor antibodies were undetectable. Significant improvement of the extraocular muscle function and resolution of the right ptosis had resulted from anticholinesterase therapy.

These findings and the clinical response to therapy were consistent with concomitant euthyroid Graves' ophthalmopathy and ocular myasthenia gravis. Coexistent isolated ocular myasthenia gravis and Graves' ophthalmopathy is rare and should be considered in patients with findings of ocular myasthenia and extraocular muscle dysfunction.

\section{Introduction}

The association between thyroid disease and myasthenia gravis is well established. ${ }^{1,2}$ Approximately 3 to $10 \%$ of myasthenia gravis patients have or will have Graves' disease ${ }^{2}$ and most of these will become thyrotoxic during follow-up. Other thyroid related abnormalities are also seen with a high frequency in patients with myasthenia gravis. ${ }^{2}$ However, euthyroid Graves' ophthalmopathy has only rarely been associated with myasthenia gravis. ${ }^{3}$ When the ocular manifestations of Graves' disease and myasthenia gravis coexist and are not associated with either overt thyroid dysfunction or generalized features of myasthenia gravis, one of the two diseases may be overlooked and diagnostic errors may stem from the overlap of clinical ocular manifestations of both disorders. We report here a case of a young woman who presented with both ocular myasthenia and euthyroid Graves' ophthalmopathy. Only careful evaluation enabled us to establish the coexistence of the two processes.

\section{Case report}

A 44 year old black female with a history of hypertension and coronary artery disease had had

Correspondence: R. Arem, M.D.

Accepted: 25 April 1990 diabetes mellitus for the past 17 years, treated with oral agents for 8 years and subsequently was begun on insulin therapy. She had a history of mild lower extremity diabetic neuropathy but no evidence of nephropathy or retinopathy.

In February 1988 she experienced a sudden onset verticle diplopia and blurred vision with discomfort in the right eye, which partially resolved over a 2-week period. One month later she noted right eye ptosis which also resolved spontaneously over the next month. The persistence of the diplopia prompted evaluation by a neurologist 3 months later, and the diagnosis of myasthenia gravis was suspected. At that time the anti-acetylcholine (anti-ACH) receptor antibodies were undetectable (normal $<0.5 \mathrm{pmol} / \mathrm{l})$. The free thyroxine index was 3.9 (normal 2.0-4.9) and a chest computed tomographic (CT) scan showed no evidence of thymoma. Edrophonium bromide (Tensilon) stimulation test was reported as negative.

In January 1989, the patient noted a left ptosis and worsening of the diplopia with a pressure sensation more severe behind the left eye and accompanied by burning and itching in both eyes. At that time CT scan of the orbits showed minimal left globe proptosis and thickening of the left medial and inferior rectus, and cerebral angiography excluded the presence of an aneurysm. Forced duction test was also interpreted as normal. 
She was admitted for evaluation at The Methodist Hospital, Houston, Texas in February 1989. The patient denied excessive sweating, palpitation, weight loss or menstrual changes, but gave a vague history of nervousness and heat intolerance. There was no family history of thyroid or muscular disease. Her physical examination revealed no evidence of tremor in the extremities, excessive moisture of the palms or onycholysis. The pupils were normally responsive to light and there was bilateral ptosis more significant on the right than the left. The left eye showed obvious proptosis and near complete ophthalmoplegia, while the extraocular movements were normal on the right, with a less significant proptosis. There was scleral and conjunctival injection, but no evidence of lid lag or globe lag. There was a small, diffuse, nontender symmetrical goitre without nodularity or bruit.

In a formal ophthalmological evaluation, the intraocular pressures were 12 and $14 \mathrm{mmHg}$ in the right and left eyes respectively with levator functions of $12 \mathrm{~mm}$ on the right and diminished to $2 \mathrm{~mm}$ on the left. Eye motility was significantly limited in all positions of gaze on the left and slightly reduced on the right. The ophthalmoscopic examination was normal. Exophthalmometry gave readings of 21 and $23 \mathrm{~mm}$ in the right and left eyes respectively (normal $<20 \mathrm{~mm}$ ). Magnetic resonance imaging (MRI) of the head confirmed the left proptosis and bilateral enlargement of the medial and inferior rectus muscles which were more prominent on the left side (see Figure 1). Forced ductions were negative on both sides. A Tensilon test showed complete resolution of ptosis and partial improvement of the eye movement on medial and lateral gaze. Thyroid function tests revealed a total thyroxine (T4) of $11.2 \mu \mathrm{g} / \mathrm{dl}$ (normal 5-12), free T4 $1.9 \mathrm{ng} / \mathrm{dl}$ (normal $1.1-2.5$ ), triiodothyronine resin uptake (T3RU) 38.7\% (normal 35-45\%), T3 $108 \mathrm{ng} / \mathrm{dl}$ (normal 90-112), free thyroid index (FTI) 10.2 (normal 5.5-11.5), thyroid stimulating immunoglobulin activity was $113 \%$ (normal $0-130$ ) and thyroid stimulating hormone (TSH) $<0.5 \mu \mathrm{U} / \mathrm{ml}$ (normal $0.5-5.5$ $\mu \mathrm{U} / \mathrm{ml})$.

The thyrotrophin releasing hormone (TRH) stimulation was flat with TSH levels less than $0.5 \mu \mathrm{U} / \mathrm{ml}$ prior to and at $30,45,60,90$ and 120 minutes following administration of $500 \mu \mathrm{g}$ TRH intravenously. The anti-microsomal and antithyroid antibodies were negative and 6-hour and 24 -hour radioactive iodine uptakes were $6.3 \%$ and $15.7 \%$ respectively. Anti- $\mathrm{ACH}$ antibodies were undetectable $(<0.3 \mathrm{pmol} / \mathrm{l})$. The erythrocyte sedimentation rate was $31 \mathrm{~mm} / \mathrm{h}$, rheumatoid factor positive at $1 / 20$ dilution and antinuclear antibody at $1 / 40$. Electromyogram (EMG) study revealed equivocal decrementing of right facial muscles with slow rate of stimulation. No change

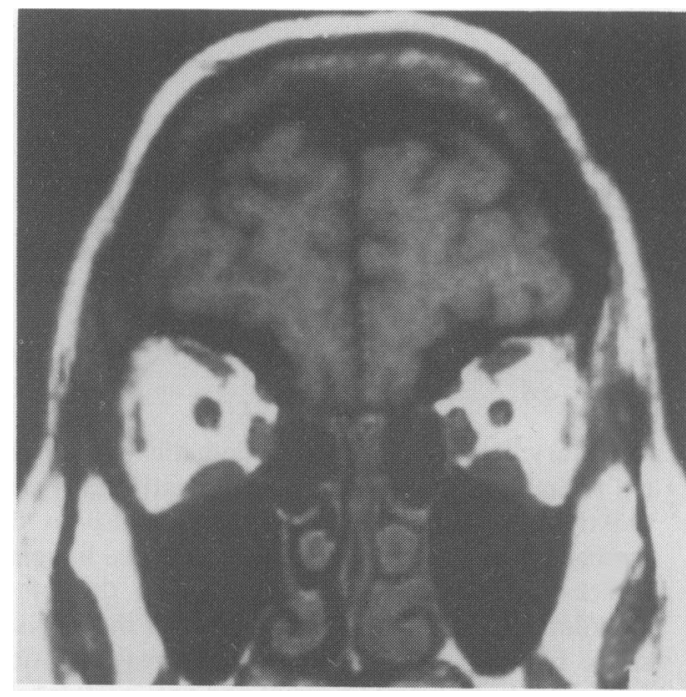

Figure 1 Magnetic resonance imaging of the orbital content showing thickening of the rectus muscles.

was found in the trapezius or hand muscles. The single fibre EMG showed a mild increase in fibre density (mean 1.5) (normal up to 1.43) and the mean consecutive difference was $30.8 \mathrm{U} / \mathrm{s}$ (normal $25 \mathrm{U} / \mathrm{s})$, consistent with a defect in neuromuscular transmission. The HLA phenotype was A28,31; B17(w4); 53(w4); DR5 (DQW7, DRw52).

The patient was begun on pyridostigmine (Mestinon), $60 \mathrm{mg}$ orally three times a day prednisone $80 \mathrm{mg}$ orally 4 times a day with complete resolution of the right ptosis and return of $60-70 \%$ of left extraocular muscle function. However, the therapy did not affect the exophthalmos and the patient continued to have verticle diplopia.

\section{Discussion}

The rarity of the co-occurrence of euthyroid Graves' disease and myasthenia gravis is not surprising because of the rarity of euthyroid Graves' ophthalmopathy itself. Ocular myasthenia gravis and euthyroid Graves' ophthalmopathy occurring at the same time has not been previously reported. In one report, a patient with multiple sclerosis was described to have an association of generalized myasthenia gravis and euthyroid Graves' disease. ${ }^{3}$ In another report, ocular myasthenia gravis occurred in a young woman who had Graves' ophthalmopathy 4 years prior to the onset of ocular myasthenia gravis and remained euthyroid until 16 years later when she became hypothyroid with evidence of elevated antithyroid antibodies consistent with Hashimoto's thyroiditis. ${ }^{1}$ Although our patient might show evidence of 
frank hyperthyroidism or generalized myasthenia gravis in the future, her current presentation is consistent with Graves' disease and myasthenia gravis with manifestations limited to the eyes.

The clinical features and extensive evaluation are in support of this association. Thus, even though the exophthalmos could have been due to myasthenia gravis, ${ }^{1}$ she had the characteristic bilateral muscle thickening, especially of the medial and inferior rectus muscle observed in Graves' ophthalmopathy but not in myasthenia gravis. In addition, the TSH response was suppressed despite clinical and biochemical euthyroidism. The coexistence of ocular myasthenia is supported by the finding of a bilateral ptosis which is not usually a feature of Graves' ophthalmopathy, by the partial response to the Tensilon test and to the pyridostigmine therapy and by the presence of abnormalities of single fibre EMG. However, the severe left ophthalmoplegia might have been due to both diseases.

In the evaluation of this patient, diagnostic difficulties did arise because of the lack of some of the characteristic features of myasthenia gravis and Graves' disease. The anti-ACH antibody titres were undetectable, the typical EMG abnormalities were absent, the forced duction test was negative and the TSI activity was negative. These characteristic features are, however, less frequently present in ocular myasthenia gravis and in euthyroid Graves' ophthalmopathy than in generalized myasthenia gravis and Graves' hyperthyroidism respectively and the absence does not refute the diagnosis. Thus, while positive anti-ACH-receptor antibody may be found in $80-90 \%$ of patients with generalized myasthenia gravis, they are only found in $17-70 \%$ of those with ocular myasthenia gravis. ${ }^{4-7}$ In addition, a typical decremental EMG response to repetitive stimulation may be undetectable in ocular myasthenia gravis. ${ }^{5,6}$ The Tensilon test which is helpful in making the diagnosis of myasthenia gravis also may be negative in ocular myasthenia gravis as ocular muscles might be refractory to the Tensilon effect. ${ }^{5,6}$

Furthermore, there is no pathognomonic biochemical or immunological marker of euthyroid Graves' disease. Thyroid stimulating immunoglobulin titre is less helpful in the diagnosis of euthyroid Graves' disease than in patients with Graves' hyperthyroidism. Solomon et al. ${ }^{8}$ found that only $50 \%$ of patients with euthyroid Graves' ophthalmopathy have elevated thyroid stimulating immunoglobulin activity, and that thyroid antibodies are less frequently found in these patients than in patients with Graves' hyperthyroidism. A more recent study also suggested that TSH binding inhibiting immunoglobulin activity, although found in all Graves' hyperthyroidism patients, was detected in only $40 \%$ of patients with euthyroid Graves' ophthalmopathy. ${ }^{9}$ Furthermore the presence of various other thyroid abnormalities such as goitre, elevated radioactive iodine uptake and abnormal T3 suppression test are not uniformly present in euthyroid Graves' disease patients. ${ }^{10,18}$ The association of certain HLA antigens with myasthenia gravis also may be different in generalized myasthenia gravis than in ocular myasthenia gravis. ${ }^{11,12}$ The HLA phenotype in our patient is different from the phenotype frequently associated with myasthenia gravis.

It is believed that both Graves' ophthalmopathy and myasthenia gravis are autoimmune diseases. The mechanism of the link between these two entities may be related to the presence of a common immunological alteration. Antibodies which bind to the eye muscle antigens were found in the sera of patients with Graves' ophthalmopathy and were called 'ophthalmopathic immunoglobulin' (OIG) ${ }^{13}$ In a recent study, however, ${ }^{14}$ OIG was found to represent a heterogeneous population of antibodies which may coincide with antibodies to myosin, actin or acetylcholine receptors. When retro-orbital muscle antigen was used, such antibodies correlated with antimyosin antibodies in euthyroid Graves' ophthalmopathy and myasthenia gravis patients. When retro-orbital muscle membrane was used as antigen, however, antibodies correlated with anti-ACH receptor antibody in the same two groups, ${ }^{14}$ suggesting an immunological link between the two processes.

In summary we report a patient who presented with concomitant Graves' ophthalmopathy and ocular myasthenia gravis who responded partially to steroids and anticholinesterase inhibitor. The association of these two processes is quite interesting and may be easily overlooked because of the overlap of the manifestations of both conditions. Awareness of this rare association may prevent overlooking a coexistent ocular myasthenia in patients with euthyroid Graves' disease. Appropriate therapy directed promptly to the myasthenia gravis may significantly alleviate some of the ocular disturbances.

\section{References}

1. Czernobilsky, H. \& Ziegler, R. Graves' ophthalmopathy, ocular myasthenia gravis and Hashimoto's thyroiditis. Isr $J$ Med Sci 1985, 21: 377-380.

2. Kiessling, W.R., Finke, R., Kotulla, P. et al. Circulating TSH-binding inhibiting immunoglobulins in myasthenia gravis. Acta Endocrinol 1982, 101: 41-46.

3. Bixenman, W.W. \& Buchsbaum, H.W. Multiple sclerosis, euthyroid restrictive Graves' ophthalmopathy and myasthenia gravis. Graefe's Arch Clin Exp Ophthalmol 1988, 226: $168-171$. 
4. Oosterhuis, H.J.G.H. The ocular signs and symptoms of myasthenia gravis. Doc Ophthalmol 1982, 52: 363-378.

5. Seybold, M.E. Myasthenia gravis. A clinical and basic science review. JAMA 1983, 250: 2516-2521.

6. Evoli, A., Tonali, P., Bartoccioni, E. \& LoMonaco, M. Ocular myasthenia diagnostic and therapeutic problems. Acta Neurol Scand 1988, 77: 31-35.

7. Garlepp, M.J., Dawkins, R.L., Christiansen, F.T. et al. Autoimmunity in ocular and generalized myasthenia gravis. $J$ Neuroimmunol 1981, 1: 325-332.

8. Solomon, D.H., Chopra, I.J., Chopra, U. \& Smith, F.J. Identification of subgroups of euthyroid Graves' ophthalmopathy. N Engl J Med 1977, 296: 181-186.

9. Kasagi, K., Hatabu, H., Tokuda, Y., Iida, Y., Endo, K. \& Konishi, J. Studies on thyrotrophin receptor antibodies in patients with euthyroid Graves' disease. Clin Endocrinol 1988, 29: 357-366.
10. Tamai, I.I. et al. Changes in thyroid functions in patients with euthyroid Graves' disease. J Clin Endocrinol Metab 1980, 50: $108-112$.

11. Christiansen, F.T., Pollack, M.S., Garlepp, M.J. \& Dawkins, R.L. Myasthenia gravis and HLA antigens in American Blacks and other races. $J$ Neuroimmunol 1984, 7: 121-129.

12. Kaichi, K. et al. Heterogeneity in myasthenia gravis: HLA phenotypes and autoantibody responses in ocular and generalized types. Ann Neurol 1987, 21: 274-278.

13. Kodama, K., Sikorska, H., Brandy-Dafoe, P., Bayly, R. \& Wall, J.R. Demonstration of a circulating autoantibody against a soluble eye muscle antigen in Graves' ophthalmopathy. Lancet 1982, ii: 1353-1356.

14. Kadlubowski, M., Irvine, W.J. \& Rowland, A.C. Anti-muscle antibodies in Graves' ophthalmopathy. J Clin Lab Immuol 1987, 24: 105-111. 